\title{
Electromagnetic Duality and New Solutions of the Non-minimally Coupled $Y(R)$-Maxwell Gravity
}

\author{
Özcan SERT* \\ Department of Mathematics, Faculty of Arts and Sciences, \\ Pamukkale University, 20017 Denizli, Türkiye
}

(Dated: October 6, 2018)

\begin{abstract}
Non-minimally coupled $Y(R)$-Maxwell gravity which have some interesting solutions may be used to understand dark matter, dark energy, the origin of cosmic magnetic field and the evaluation of the universe. We give some new solutions to the model such as spherically symmetric electric, magnetic and dyon solutions. We point out an existence of an electromagnetic duality transformation for the model.
\end{abstract}

\footnotetext{
* osert@pau.edu.tr
} 


\section{INTRODUCTION}

The non-minimally coupled $Y(R)$-Maxwell models [1 18] have some good properties for shedding light on some concepts, such as magnetization in matter, cosmic magnetic fields, dark matter, dark energy and the evaluation of the universe. If one looks for a new fundamental law in order to explain these concepts, its best implication is to have new symmetries. Symmetries have an important role in physics and mathematics. Because they imply an existence of a corresponding fundamental conservation law and dictate the form of the law. Thus the recent theoretical explorations are mostly based on the search for new symmetries of nature. Thus, duality symmetry of the field equations of the $Y(R)$-Maxwell models is the main motivation of this study.

The duality symmetry of Einstein-Maxwell field equations has received much attention in gauge theories and gravitation. It provides useful tools to obtain new solutions to the field equations for studying different regimes of the theory. According to this symmetry, the Maxwell equations $d F=0$ and $d * F=0$ remain invariant under the duality transformation $(E, B) \rightarrow(B,-E)$ or $(F, * F) \rightarrow(* F,-F)$. But, the Maxwell Lagrangian changes sign under the transformation. That is, this symmetry is valid only in the field equations, not Lagrangian. This duality (Heaviside duality) of the electromagnetic system provides a connection between the electric and the magnetic black hole solutions. Then if we know an electrically charged solutions, we can find also corresponding magnetically charged solutions and vice verse. The duality transformation of electromagnetic fields are related to the conserved quantities such as currents and helicity.

In this study we show that the non-minimal theory has a new duality transformation which is $(F, * F) \rightarrow(* G,-G)$ and $Y \rightarrow 1 / Y(\mathrm{G}$ is excitation 2-form). In this case, similar to the minimal Einstein-Maxwell theory the field equations remain invariant and the nonminimal part of the Lagrangian changes sign under the transformation, and it becomes a different model. While this duality transformation provides a connection between the electric and the magnetic black hole solutions for the non-minimal model, additionally, it determines the structure of the non-minimal coupling function $Y(R)$.

In the present paper, we proceed to investigate the non-minimal couplings of gravitational and electromagnetic fields giving a new duality transformation for the model. We obtain some new dyon solutions with electric and magnetic field. Then, we point out that the 
solutions and the field equations are left invariant under the new duality transformation.

\section{FIELD EQUATIONS OF THE NON-MINIMALLY COUPLED THEORY AND DUALITY TRANSFORMATIONS}

We obtain the field equations of the non-minimal theory by a variational principle from an action

$$
I\left[e^{a}, \omega_{b}^{a}, F\right]=\int_{M} L
$$

where $\left\{e^{a}\right\}$ and $\left\{\omega^{a}{ }_{b}\right\}$ are the fundamental gravitational field variables and $F=d A$ is the electromagnetic field 2-form. The space-time metric $g=\eta_{a b} e^{a} \otimes e^{b}$ has the signature $(-+++)$ and we fix the orientation by setting $* 1=e^{0} \wedge e^{1} \wedge e^{2} \wedge e^{3}$. Torsion 2-forms $T^{a}$ and curvature 2 -forms $R_{b}^{a}$ are given as

$$
\begin{gathered}
T^{a}=d e^{a}+\omega_{b}^{a} \wedge e^{b}, \\
R_{b}^{a}=d \omega_{b}^{a}+\omega_{c}^{a} \wedge \omega_{b}^{c} .
\end{gathered}
$$

We start with the following Lagrangian density 4-form;

$$
L=\frac{1}{2 \kappa^{2}} R * 1-\frac{1}{2} Y(R) F \wedge * F+T^{a} \wedge \lambda_{a},
$$

where $\kappa^{2}=8 \pi G$ is Newton's universal gravitational constant $(c=1)$ and $R$ is the curvature scalar which can be found by applying interior product $\iota_{a}$ twice to the curvature tensor $R_{a b}$ 2-form. This Lagrangian density involves Lagrange multiplier 2-form $\lambda_{a}$ whose variation imposes the zero-torsion constraint $T^{a}=0$. Then we use only the unique metriccompatible Levi-Civita connection. We use the shorthand notation $e^{a} \wedge e^{b} \wedge \cdots=e^{a b \cdots}$, and $\iota_{a} F=F_{a}, \quad \iota_{b a} F=F_{a b}, \quad \iota_{a} R_{b}^{a}=R_{b}, \quad \iota_{b a} R^{a b}=R$. The field equations are obtained by considering the independent variations of the action with respect to $\left\{e^{a}\right\},\left\{\omega^{a}{ }_{b}\right\}$ and $\{F\}$. The electromagnetic field components are read from the expansion $F=\frac{1}{2} F_{a b} e^{a} \wedge e^{b}$.

After taking the infinitesimal variations of the total Lagrangian density $L$, we find the Einstein and Maxwell field equations [16, 17] for the extended theory as

$$
\begin{aligned}
& \frac{1}{2 \kappa^{2}} R^{b c} \wedge * e_{a b c}+\frac{1}{2} Y\left(\iota_{a} F \wedge * F-F \wedge \iota_{a} * F\right)+Y_{R}\left(\iota_{a} R^{b}\right) \iota_{b}(F \wedge * F) \\
& +\frac{1}{2} D\left[\iota^{b} D\left(Y_{R} F_{m n} F^{m n}\right)\right] \wedge * e_{a b}=0,
\end{aligned}
$$




$$
d(* Y F)=0, \quad d F=0 .
$$

where $Y_{R}=\frac{d Y}{d R}$. One can show that the action (44) and the field equations (15)-(16) when written out explicitly in any local coordinate system are equivalent to those given by Bamba and Odintsov[14].

The non-minimal coupling model modifies both the Maxwell and the Einstein field equations. Modifications to the Maxwell equations can be associated with the magnetization and the polarization of a specific medium, while modifications to the Einstein equations changes the space-time metric. Here the medium can be considered as containing both magnetically and electrically polarisable matter with $G=Y F$. Now we want to show that these field equations (5)-(6) remain invariant under the duality transformation $(F, * F) \rightarrow(* G,-G)$ and $Y \rightarrow 1 / Y$. As we see from the following steps, each part of the field equations (5) remain invariant;

$$
\begin{gathered}
Y\left(\iota_{a} F \wedge * F-F \wedge \iota_{a} * F\right) \rightarrow\left(\frac{1}{Y}\right)\left[\iota_{a} * G \wedge(-G)-* G \wedge \iota_{a}(-G)\right] \\
=Y\left(\iota_{a} F \wedge * F-F \wedge \iota_{a} * F\right) \\
Y_{R}\left(\iota_{a} R^{b}\right) \iota_{b}(F \wedge * F) \rightarrow\left(-\frac{1}{Y^{2}}\right) Y_{R}\left(\iota_{a} R^{b}\right) \iota_{b}[* G \wedge(-G)] \\
=Y_{R}\left(\iota_{a} R^{b}\right) \iota_{b}(F \wedge * F) \\
\frac{1}{2} D\left[\iota^{b} D\left(Y_{R} F_{m n} F^{m n}\right)\right] \wedge * e_{a b} \rightarrow \frac{1}{2} D\left[\iota^{b} D\left(\left(-1 / Y^{2}\right) Y_{R}(* G)_{m n}(* G)^{m n}\right)\right] \wedge * e_{a b} \\
=\frac{1}{2} D\left[\iota^{b} D\left(Y_{R} F_{m n} F^{m n}\right)\right] \wedge * e_{a b}
\end{gathered}
$$

where $\iota_{n} \iota_{m} * G=(* G)_{m n}=Y(* F)_{m n}$. In the last step we have used the fact that $(* F)_{m n}(* F)^{m n}=-F_{m n} F^{m n}$. It is obvious that the first part of (5) and the Maxwell equations (6) do not change under this transformation. But, the non-minimal part of the Lagrangian density changes sign under the transformation as it is in the minimal EinsteinMaxwell theory;

$$
F \wedge * Y F \rightarrow * G \wedge \frac{1}{Y}(-G)=-F \wedge * Y F
$$


Thus this transformation leads to a different dual model. If we write the explicit form of $F$ in components as

$$
F=E_{1} e^{01}+E_{2} e^{02}+E_{3} e^{03}+B_{1} e^{23}-B_{2} e^{13}+B_{3} e^{12}
$$

then we calculate $* G$

$$
* G=Y B_{1} e^{01}+Y B_{2} e^{02}+Y B_{3} e^{03}-Y E_{1} e^{23}+Y E_{2} e^{13}-Y E_{3} e^{12} .
$$

Thus the transformation $(F, * F) \rightarrow(* G,-G)$ corresponds to $\left(E_{i}, B_{i}\right) \rightarrow\left(Y B_{i},-Y E_{i}\right)$ in terms of electric $E_{i}$ and magnetic $B_{i}$ components of electromagnetic tensor $F, i=1,2,3$. Thus we find a map between electric and magnetic solutions in the electrically and magnetically polarizable medium.

\section{SOME STATIC, SPHERICALLY SYMMETRIC SOLUTIONS}

We consider (1+3)-dimensional static, spherically symmetric solutions to the non-minimal model which are given by the metric

$$
g=-f(r)^{2} d t^{2}+f(r)^{-2} d r^{2}+r^{2} d \theta^{2}+r^{2} \sin (\theta)^{2} d \phi^{2}
$$

\section{A. Magnetic Monopole Solutions}

In order to show the electromagnetic duality explicitly in solutions and in field equations, first we take the electromagnetic tensor $F$ which has only the magnetic component $B=B_{1}$;

$$
F=B(r) r^{2} \sin (\theta) d \theta \wedge d \phi=B(r) e^{2} \wedge e^{3}
$$

The field equations (5) and (6) give us the following system of equations [15]:

$$
\begin{aligned}
& \frac{1}{\kappa^{2}}\left(\frac{f^{2^{\prime}}}{r}+\frac{f^{2}-1}{r^{2}}\right)+Y_{R} B^{2}\left(\frac{f^{2^{\prime \prime}}}{2}+\frac{f^{2^{\prime}}}{r}\right)+\frac{1}{2} Y B^{2}+\left[\left(B^{2} Y_{R}\right)^{\prime} f\right]^{\prime} f+\frac{2}{r} f^{2}\left(B^{2} Y_{R}\right)^{\prime}=0 \\
& \frac{1}{\kappa^{2}}\left(\frac{f^{2^{\prime}}}{r}+\frac{f^{2}-1}{r^{2}}\right)+Y_{R} B^{2}\left(\frac{f^{2^{\prime \prime}}}{2}+\frac{f^{2^{\prime}}}{r}\right)+\frac{1}{2} Y B^{2}+\left(B^{2} Y_{R}\right)^{\prime}\left(\frac{f^{2}}{2}+\frac{2 f^{2}}{r}\right)=0 \\
& \frac{1}{\kappa^{2}}\left(\frac{f^{2^{\prime \prime}}}{2}+\frac{f^{2^{\prime}}}{r}\right)+Y_{R} B^{2}\left(\frac{f^{2^{\prime}}}{r}+\frac{f^{2}-1}{r^{2}}\right)-\frac{1}{2} Y B^{2}+\left[\left(B^{2} Y_{R}\right)^{\prime} f\right]^{\prime} f+\left(B^{2} Y_{R}\right)^{\prime}\left(\frac{f^{2^{\prime}}}{2}+\frac{f^{2}}{r}\right)=0 \\
& B=\frac{q_{m}}{r^{2}}
\end{aligned}
$$


Here the curvature scalar is calculated as

$$
R=-f^{2^{\prime \prime}}-\frac{4}{r} f^{2^{\prime}}-\frac{2}{r^{2}}\left(f^{2}-1\right)
$$

The following solutions to these differential equations are found in [15] for some non-minimal coupling functions. That is;

$$
\begin{aligned}
f^{2}(r) & =1-\frac{2 M}{r}+\frac{a_{1} \kappa^{2} q^{2}}{r^{2}} \ln \frac{r}{r_{0}}+\frac{\kappa^{2} q^{2}\left(1+5 a_{1}\right)}{4 r^{2}}, \quad \text { for } a_{1} \neq 0, \\
B(r) & =\frac{q_{m}}{r^{2}}
\end{aligned}
$$

for

$$
Y(R)=1-a_{1} \ln \left(\frac{R}{R_{0}}\right)
$$

and

$$
\begin{aligned}
f^{2}(r) & =1-\frac{c}{r}+\frac{\kappa^{2} q^{2}}{4 r^{2}}-\frac{a_{2}(\beta-1)^{2}}{4 \beta(3 \beta+1)} r^{\frac{2 \beta+2}{\beta-1}}, \quad \text { for } R_{0} \neq 0, \quad \beta \neq 0,1,-\frac{1}{3} \\
B(r) & =\frac{q_{m}}{r^{2}},
\end{aligned}
$$

for

$$
Y(R)=1-\left(\frac{R}{R_{0}}\right)^{\beta}
$$

where $q_{m}$ is determined by the Gauss integral

$$
\frac{1}{4 \pi} \int_{S^{2}} F=\frac{1}{4 \pi} \int_{S^{2}} B(r) r^{2} \sin \theta d \theta \wedge d \phi=q_{m}
$$

To show the existence of the electromagnetic duality in these non-minimally coupled models, we compare these magnetic solutions with the previous electric solutions. These equations we found in (15) and (16) for the magnetic field turn out to be the equations given in Ref. [16] (eqs. (23)-(26)) for an electric field ansatz under these transformations $B \rightarrow-D_{e}$, $q_{m} \rightarrow-q_{e}$ and $Y \rightarrow \frac{1}{Y}$. Here the displacement field is defined $D_{e}=Y E$ (see Ref. [16, 17] taking $\left.G=Y F=Y E e^{01}\right)$. Consequently the above solutions turn out to be the solutions given in Ref. [16, 17]. Thus we reach the previous different model. 


\section{B. Dyon Solutions}

In this subsection our aim is to find new solutions and to show their electromagnetic duality for the non-minimal model. Now we consider the electromagnetic field of a dyon which has the magnetic and electric components;

$$
F=E(r) d t \wedge d r+B(r) r^{2} \sin (\theta) d \theta \wedge d \phi=E(r) e^{0} \wedge e^{1}+B(r) e^{2} \wedge e^{3}
$$

We calculate the field equations for the metric (13) together with the dyon electromagnetic field 2-form (25). Then we find

$$
\begin{aligned}
\frac{1}{\kappa^{2}}\left(f^{2^{\prime \prime}}-\frac{2}{r^{2}}\left(f^{2}-1\right)\right)-Y E^{2}-Y B^{2} & =0, \\
Y E & =\frac{q_{e}}{r^{2}}, \\
B & =\frac{q_{m}}{r^{2}} .
\end{aligned}
$$

under the constrain

$$
Y_{R}\left(E^{2}-B^{2}\right)=\frac{1}{\kappa^{2}}
$$

We emphases that these differential equations (26)-(29) are invariant under the duality transformations $(E, B) \rightarrow(Y B,-Y E),\left(q_{e}, q_{m}\right) \rightarrow\left(q_{m},-q_{e}\right)$ and $Y \rightarrow 1 / Y$.

Additionally, one can look for a non-minimal function for a useful gravitational metric. Now we consider the following metric function with an extra logarithmic term

$$
f^{2}(r)=1-\frac{C_{1}}{r}-C_{3} \ln (r)
$$

which is viable to obtain the constant velocity of the trajectories for large values of the radial

distance [19]. They also comes from the computation of a running gravitational coupling as a result of the quantum effects [20] and the TeVeS theory [21, 22]. Here $C_{1}$ and $C_{3}$ are constants. Then we determine the structure of the non-minimal function $Y$ which is consistent with the solution. After solving the field equations for the model, we find the non-minimal function, the electric and magnetic fields;

$$
\begin{aligned}
Y(r)= & \frac{C_{3}}{2 q_{m}^{2} \kappa^{2}} r^{2}+\frac{C_{3}}{q_{m}^{2} \kappa^{2}} r^{2} \ln (r) \\
& -\frac{1}{2 q_{m}^{2} \kappa^{2}} \sqrt{C_{3}^{2} r^{4}+4 C_{3}^{2} r^{4} \ln (r)(1+\ln (r))-4 \kappa^{4} q_{e}^{2} q_{m}^{2}} \\
B(r)= & \frac{q_{m}}{r^{2}} \\
E(r)= & \frac{q_{e}}{Y(r) r^{2}} .
\end{aligned}
$$


We note that the dependence of $Y$ to $R$ is not explicit.

In order to find asymptotically flat solutions with an explicit $Y(R)$ function, we consider

$$
f^{2}(r)=1-\frac{2 M}{r}+\frac{C_{2}}{r^{2}}-\frac{C_{3} \ln (r)}{r}
$$

then for $C_{3} \neq 0$ we obtain

$$
\begin{aligned}
Y(r) & =\frac{2 C_{2}}{q_{m}^{2} \kappa^{2}}+\frac{3 C_{3}}{2 q_{m}^{2} \kappa^{2}} r-\frac{1}{2 q_{m}^{2} \kappa^{2}} \sqrt{16 C_{2}^{2}-4 q_{e}^{2} \kappa^{4} q_{m}^{2}+24 C_{2} C_{3} r+9 C_{3}^{3} r^{2}} \\
B(r) & =\frac{q_{m}}{r^{2}} \\
E(r) & =\frac{q_{e}}{Y(r) r^{2}} .
\end{aligned}
$$

We can solve $r$ form $R(r)=\frac{C_{3}}{r^{3}}$, thus we can find $Y(R)$ :

$$
Y(R)=\frac{2 C_{2}}{q_{m}^{2} \kappa^{2}}+\frac{3 C_{3}^{\frac{4}{3}}}{2 q_{m}^{2} \kappa^{2}} R^{-\frac{1}{3}}-\frac{1}{2 q_{m}^{2} \kappa^{2}} \sqrt{16 C_{2}^{2}-4 q_{e}^{2} \kappa^{2}+24 C_{2} C_{3}^{\frac{4}{3}} R^{-\frac{1}{3}}+9 C_{3}^{\frac{8}{3}} R^{-\frac{2}{3}}}
$$

Lastly, we can find the solutions with an extra correction power law term;

$$
f^{2}(r)=1+\frac{C_{1}}{r}-C_{3} r^{n}
$$

which is a candidate to solve both the dark energy and dark matter problem [23].

$$
\begin{aligned}
Y(r)= & \frac{C_{3}}{2 q_{m}^{2} \kappa^{2}}\left(n^{2}-n-2\right) r^{n+2} \\
& -\frac{1}{2 q_{m}^{2} \kappa^{2}} \sqrt{C_{3}^{2}\left(n^{4}-2 n^{3}-3 n^{2}+4 n+4\right) r^{2 n+4}-4 \kappa^{4} q_{e}^{2} q_{m}^{2}} \\
B(r)= & \frac{q_{m}}{r^{2}} \\
E(r)= & \frac{q_{e}}{Y(r) r^{2}}
\end{aligned}
$$

Here we note that one can consider the case $q_{e}=0$ in these solutions. Thus one can reach magnetically charged solutions. This means that we have also corresponding electrically charged solutions because of the duality transformation.

\section{CONCLUSION}

We have investigated the duality transformation of the $Y(R)$-Maxwell theory of gravity and new solutions of it. We find firstly a new duality transformation which leave invariant the field equations, while the non-minimal part of the Lagrangian changes sign under that transformation, and this leads to a different model. This duality transformation is a map 
between the electromagnetic field $F$ with $* G=* Y(R) F$ under which all the non-minimal gravitational solutions are mapped into new solutions. Also it determines a relation about how the non-minimal coupling function $Y(R)$ transforms in the specific medium. Thus we can find new solutions and categorize them in order to gain more insights on the electric and magnetic fields in the medium. We give some examples to the duality which transforms spherically symmetric, magnetic solutions to the electric solutions in the electrically and magnetically polarizable medium. After then we find various non-minimal functions of the theory for some interesting spherically symmetric metric functions [19 23] with the magnetic and electric charge. Finally we show that these solutions also satisfy this duality.

\section{ACKNOWLEDGEMENT}

This work was supported by Pamukkale University (BAP Project No: 2012BSP014).

\section{REFERENCES}

[1] A. R. Prasanna, Phys. Lett. A 37, 337 (1971)

[2] G. W. Horndeski, J. Math. Phys. 17, 1980 (1976)

[3] I. T. Drummond, S. J. Hathrell, Phys. Rev. D 22, 343 (1980)

[4] H. A. Buchdahl, J. Phys. A 12, 1037 (1979)

[5] T. Dereli, G. Üçoluk, Class. Q. Grav. 7, 1109 (1990).

[6] F. Müller-Hoissen, Class. Q. Grav. 5, L35 (1988)

[7] A. B. Balakin, J. P. S. Lemos, Class. Q. Grav. 22, 1867 (2005)

[8] A.B. Balakin, H. Dehnen, A.E. Zayats, Phys. Rev. D 79 024007, (2009)

[9] A.B. Balakin and A.E. Zayats, Gravitation and Cosmology 14, 86-94, (2008)

[10] T. Dereli, Ö. Sert, Phys. Rev. D 83, 065005 (2011)

[11] G. Lambiase, S. Mohanty and G. Scarpetta, JCAP 07, 019, (2008)

[12] Y. Liu and J. Jing, Gen. Relativ. Gravit., 44, 7 (2012)

[13] K. Bamba and S. D. Odintsov, JCAP 0804, 024, (2008) 
[14] K. Bamba, S. Nojiri and S. D. Odintsov, JCAP, 0810, 045, (2008)

[15] Ö. Sert, Eur. Phys. J. Plus 127: 152 (2012)

[16] T. Dereli, Ö. Sert, Eur. Phys. J. C 71, 3, 1589 (2011)

[17] T. Dereli, Ö. Sert, Mod. Phys. Lett. A 26, 20, 1487-1494 (2011)

[18] Ö. Sert and M. Adak, "An anisotropic cosmological solution to the Maxwell- $Y(R)$ gravity", arXiv:1203.1531 [gr-qc]

[19] J.C. Fabris, J. P. Campos Gen. Relativ. Gravit., 41, 1 (2009)

[20] I.L. Shapiro, J. Sola and H. Stefancic, JCAP, 01, 012 (2005)

[21] J. Bekenstein, Phys. Rev. D 70, 3509 (2004)

[22] J.Ph., Bruneton, G., Esposito-Farese, Phys. Rev. D 76, 124012 (2007)

[23] S., Capozziello, V.F., Cardone, A. Troisi, Mon. Not. Roy. Astron. Soc. , 3754 (2007) 